\title{
Desenvolvimento de uma ferramenta computacional de análise de hidrogramas
}

\author{
Tomas Carlotto
}

Universidade Federal da Fronteira Sul, UFFS, Erechim, RS

Pedro Luiz Borges Chaffe ${ }^{2}$

Departamento de Engenharia Sanitária e Ambiental, UFSC, Florianópolis, SC

Roberto Valmir da Silva ${ }^{3}$

Universidade Federal da Fronteira Sul, UFFS, Erechim, RS

\section{José Mario Vicensi Grzybowski ${ }^{4}$}

Universidade Federal da Fronteira Sul, UFFS, Erechim, RS

\begin{abstract}
Resumo. Este trabalho trata da elaboração de uma ferramenta computacional para extração de coeficientes característicos de bacias hidrográficas a partir de análises de hidrogramas. As aplicações desta ferramenta são destinadas à: realizar de separação de escoamentos por filtros numéricos; determinação do início e fim de períodos de ascensão e recessão com a utilização de métodos de aproximação diferencial; criação de Curva Mestra de Recessão (MRC) pela sobreposição de múltiplas curvas de recessão e extração dos coeficientes da MRC para caracterizar bacias hidrográficas.
\end{abstract}

Palavras-chave. Hidrologia, Hidrogramas, Filtros numéricos, MRC

\section{Introdução}

Este trabalho expõe a elaboração de uma ferramenta computacional para extração de coeficientes característicos de bacias hidrográficas a partir de análises de hidrogramas com objetivo de facilitar a compreensão da dinâmica das águas subterrâneas e superficiais em escala local e regional. A ferramenta oferece recursos para estudar a separação de escoamentos pela utilização de filtros numéricos $[1,2,4]$ que podem ser facilmente aplicáveis e retornam resultados satisfatórios, podendo ser utilizados em casos onde as aplicações de métodos empíricos com o uso de traçadores [5] tornam-se inviáveis. A

\footnotetext{
${ }^{1}$ thomas.carl@hotmail.com

2 pedro.chaffe@ufsc.br

3 roberto.silva@uffs.edu.br

4 jose.grzybowski@uffs.edu.br
} 
separação dos escoamentos permite fazer comparações entre os quantitativos dos escoamentos totais e subterrâneos e extrair o índice de fluxo de base (BFI) importante para estimar a recarga das águas subterrâneas. Além dos métodos de separação de escoamentos a ferramenta permite fazer outros tipos de análises direcionadas ao estudo das recessões e recargas das águas subterrâneas como forma de caracterizar bacias hidrográficas. Para a realização destas análises desenvolveu-se um módulo de criação de curvas mestras de recessão (MRC) que realiza sobreposição de todas as curvas de recessão que compõe o hidrograma o que resulta na formação de uma única curva (MRC) característica dos períodos recessão da bacia [4]. Outra funcionalidade da ferramenta é a de permitir o estudo direto das recessões para cada evento. Estas duas aplicações permitem determinar o coeficiente de recessão $(\alpha)$ utilizado para caracterizar bacias hidrográficas e também para estabelecer parâmetros de entrada para os filtros numéricos de separação de escoamentos. Quando o coeficiente de recessão é obtido pela MRC tem-se um coeficiente característico da bacia que pode ser usado para estimar a capacidade do escoamento subterrâneo em manter os corpos hídricos durante períodos de estiagem. Para a criação da ferramenta computacional de análise de hidrogramas utilizou-se o software MATLAB versão R2013a. Como resultado obteve-se uma ferramenta que possibilita o estudo de hidrogramas de forma otimizada.

\section{Conclusão}

A ferramenta desenvolvida facilita o acesso à métodos de grande aplicação em estudos hidrológicos de caracterização de bacias hidrográficas, e contribui com um recurso computacional que ficará disponível para o uso e para o desenvolvimento de novas funcionalidades.

\section{Referências}

[1] T. G. Chapman, Comment on "Evaluation of Automated Techniques for Base Flow and Recession Analyses" by R. J. Nathan and T. A. McMahon, Water Resources Research, vol. 27, 1783-1784, (1991).

[2] K. Eckhardt, How to construct recursive digital filters for baseflow separation, Hydrological Processes, vol. 19, 507-515, (2005).

[3] R. Lamb and K. J. Beven, Using interactive recession curve analysis to specify a general catchment storage model, Hydrology and Earth System Sciences, vol. 1, 101113, (1997).

[4] V. Lyne and M. Hollick, Stochastic time-variable rainfall-runoff modelling, Natl. Conf. Publ. Inst. Eng. Aust., vol. 10, 89-92, (1979).

[5] K. J. Mcguire and J. J. Mcdonnell, A review and evaluation of catchment transit time modeling, Journal of Hydrology, vol. 330, 543-563, (2006). 